\title{
Antagonism of tumour necrosis factor $\alpha$ in refractory asthma
}

\section{Mike A Berry, ${ }^{1}$ lan D Pavord ${ }^{2}$}

Asthma that is refractory to treatment with inhaled corticosteroids affects $5-10 \%$ of patients with asthma; it is an important health and economic problem in the UK and most industrialised nations. It is estimated that $€ 17.7$ billion is spent on asthma healthcare per annum in Europe, ${ }^{1}$ of which a disproportionately large amount is attributed to patients with refractory asthma, on average six times more per patient compared with mild to moderate disease. ${ }^{2}$ Patients with this condition suffer considerable morbidity and mortality and they therefore represent an important unmet clinical need. The European Respiratory Society ${ }^{2}$ and the American Thoracic Society ${ }^{3}$ have identified research into the mechanisms and treatment of refractory asthma as a research priority.

Asthma can be apparently refractory to treatment because of poor treatment adherence or because symptoms are caused by comorbid conditions such as rhinitis, hyperventilation and bronchiectasis. ${ }^{4}$ However, a significant number of patients have genuinely severe disease. The patterns of refractory disease are heterogeneous, both between and within patients: some have recurrent exacerbations with satisfactory day-to-day control of symptoms and lung function; in others the predominant feature is symptoms due to persistent airway dysfunction. There is also heterogeneity in the response to treatment, with some patients not achieving control despite maximum corticosteroid therapy and others achieving control, but requiring an unacceptably high dose systemic corticosteroid treatment to do so. We are only beginning to understand how these different clinical patterns of disease relate to airway dysfunction and the pattern of airway inflammation. The ultimate hope is that better understanding of refractory asthma phenotypes might allow additional treatment to be better targeted. This is important, as treatment options are often expensive

\footnotetext{
${ }^{1}$ University of Birmingham, Edgbaston, Birmingham, UK; ${ }^{2}$ Institute for Lung Health, University Hospitals of Leicester NHS Trust, Glenfield Hospital, Leicester, UK

Correspondence to: Dr Mike A Berry, University of Birmingham, Edgbaston, Birmingham B15 2TT, UK; m.a.berry@bham.ac.uk
}

and potentially toxic. Until treatment can be targeted effectively and consistently across multiple sites, we should be prepared for the frustrating roller coaster of initial encouraging results from single centre studies of small numbers of well characterised patients followed by disappointing results from larger multicentre studies. The anti-tumour necrosis factor $\alpha$ $(\mathrm{TNF} \alpha)$ story in refractory asthma is a good example of this.

The rationale for investigating the role of $\mathrm{TNF} \alpha$ in refractory asthma is strong. Firstly, many of the pathophysiological abnormalities that set refractory asthma apart from more easily controllable disease, including increased neutrophilic airway inflammation, exaggerated airway remodelling, corticosteroid resistance, fixed airflow obstruction and systemic inflammation, can be explained on the basis of increased TNF $\alpha$ production within the airway. ${ }^{56}$ Secondly, factors associated with the development of refractory asthma such as smoking, ${ }^{7}$ endotoxin exposure, ${ }^{8}$ obesity ${ }^{9}$ and chronic infection ${ }^{10}$ are associated with activation of the innate immune response and therefore might be expected to increase airway $\mathrm{TNF} \alpha$ production. Thirdly, there is consistent evidence that TNF $\alpha$ is overexpressed in refractory asthma compared with mild to moderate asthma, not only within the airway ${ }^{11}$ but also systemically. ${ }^{12}$ Fourthly, administration of inhaled recombinant TNF $\alpha$ leads to airway hyperresponsiveness in patients with asthma ${ }^{13}$ and in normal controls, ${ }^{14}$ possibly via a direct effect on airway smooth muscle ${ }^{15}$ or associated mast cells. ${ }^{16}$ The final impetus for investigating the TNF $\alpha$ axis in refractory asthma is that it has become practical to do so with the advent of a number of antagonists, which have been shown to be effective and reasonably safe in other diseases associated with TNF $\alpha$ overproduction.

Initial experience with anti-TNF $\alpha$ treatment of refractory asthma was very encouraging. In an initial open label, 12 week study ${ }^{17}$ and a subsequent 10 week double blind, randomised, placebo controlled, crossover study of 10 subjects, ${ }^{18}$ treatment with etanercept was associated with a marked reduction in methacholine airway responsiveness, improved symptoms, better quality of life scores and improved lung function. The findings in less severe asthma have been less compelling. In a double blind, placebo controlled, parallel group design study of 38 patients, treatment with infliximab for 12 weeks resulted in a decrease in the number of patients with mild deteriorations in asthma control compared with placebo, but there was no effect on the primary endpoint, change in morning peak expiratory flow. ${ }^{19}$ No major safety issues were raised in the three studies and there was considerable enthusiasm to extend this work to multicentre studies of larger populations employing more patient relevant outcomes.

One such study is reported by Morjaria and colleagues ${ }^{20}$ in this issue of Thorax (see page 584). They carried out a randomised, placebo controlled, parallel group study of 12 weeks of treatment with etanercept in 39 subjects with refractory asthma. Treatment was associated with a significant reduction in the Juniper Asthma Control Score, reflecting better asthma control, but no significant effect on asthma related quality of life. There was no effect of treatment on airway responsiveness or lung function nor was there a clinically important effect on measures of eosinophilic and neutrophilic airway inflammation.

Why are the findings of this study so much less compelling than the findings of the initial pilot studies? The population studied by Morjaria and colleagues ${ }^{20}$ had much less severe airway hyperresponsiveness than the population studied in the pilot studies and it is possible that less clear evidence of benefit was seen because the beneficial effects of TNF $\alpha$ antagonism is greater in those with more severe or more corticosteroid resistant airway dysfunction. Another possibility is that the population studied by Morjaria et al contained a higher proportion of patients whose refractory symptoms were caused by non-asthma related factors. In support of this, a post hoc analysis suggested that the beneficial effects of treatment on asthma control and quality of life was more marked when the analysis was restricted to the 29 subjects not taking antidepressants. Finally, it is important to consider whether the arbitrary and sometimes illogical exclusion criteria employed in many industry sponsored multicentre studies result in loss of the patients who are most likely to respond to treatment. 
What then can we learn from this story and how can we move the field of new drug development in refractory asthma forward? One obvious but important and often overlooked priority is to study the correct patient population and choose the right outcome measure. Thus if one is investigating an anti-eosinophil treatment, it makes sense to begin investigation in a population with severe eosinophilic airway inflammation and choose an inflammation related outcome measure such as exacerbation frequency. In contrast, if the major effects of the investigational agent are on airway smooth muscle function (as seems to be the case with anti-TNF $\alpha$ treatment), then a better population would be those with severe and corticosteroid resistant airway dysfunction, and better outcome measures would be symptoms, variable airflow obstruction and airway hyperresponsiveness. Another important priority is to identify biomarkers of treatment response. In our initial study the beneficial effects of etanercept were closely related to peripheral blood mononuclear cell membrane TNF $\alpha$ expression. ${ }^{21}$ Whether this biomarker can be used to identify a population where anti-TNF $\alpha$ treatment can be used effectively and safely should be investigated.

At a later stage in development, or if the main effects of the investigational agent are unclear, then it is important to evaluate a sufficiently large and well characterised population for relationships between baseline demographics, potential biomarkers and treatments response to be identified. Considerable thought should be put into recruitment criteria with the emphasis more on identifying and including the at need population and less on recruiting a pure population based on arbitrary clinical and physiological criteria. If we can do this, we will learn more about the pathophysiology and heterogeneity of refractory asthma and we may have more to offer a group of patients who currently have considerable unmet needs.

\section{Competing interests: None.}

Thorax 2008;63:571-572. doi:10.1136/thx.2007.095042

\section{REFERENCES}

1. Loddenkemper R, Gibson GJ, Sibille Y. Asthma burden. In: Loddenkemper R, ed. European lung white book. Sheffield: European Respiratory Society, 2003:16-25.

2. Chung KF, Godard P, Adelroth E, et al. Difficult/ therapy-resistant asthma: the need for an integrated approach to define clinical phenotypes, evaluate risk factors, understand pathophysiology and find novel therapies. ERS Task Force on Difficult/TherapyResistant Asthma. European Respiratory Society. Eur Respir J 1999;13:1198-208.

3. American Thoracic Society. Proceedings of the ATS workshop on refractory asthma: current understanding, recommendations, and unanswered questions. Am J Respir Crit Care Med 2000;162:2341-51

4. Heaney LG, Robinson DS. Severe asthma treatment: need for characterising patients. Lancet 2005;365:974-6

5. Heffler E, Berry M, Pavord ID. Tumor necrosis factoralpha: a promising therapeutic target for asthma? BioDrugs 2007;21:345-9.

6. Cazzola M, Polosa R. Anti-TNF-alpha and Th1 cytokinedirected therapies for the treatment of asthma. Curr Opin Allergy Clin Immunol 2006;6:43-50.

7. Churg A, Dai J, Tai H, et al. Tumor necrosis factoralpha is central to acute cigarette smoke-induced inflammation and connective tissue breakdown. Am J Respir Crit Care Med 2002;166:849-54

8. O'Malley WE, Achinstein B, Shear MJ. Journal of the National Cancer Institute, Vol. 29, 1962: Action of bacterial polysaccharide on tumors. II. Damage of sarcoma 37 by serum of mice treated with Serratia marcescens polysaccharide, and induced tolerance. Nutr Rev 1988:46:389-91.

9. Hotamisligil GS. The role of TNFalpha and TNF receptors in obesity and insulin resistance. $J$ Intern Med 1999;245:621-5

10. Reed CE, Milton DK. Endotoxin-stimulated innate immunity: A contributing factor for asthma. J Allergy Clin Immunol 2001;108:157-66.

11. Howarth PH, Babu KS, Arshad HS, et al. Tumour necrosis factor (TNFalpha) as a novel therapeutic target in symptomatic corticosteroid dependent asthma. Thorax 2005;60:1012-18.

12. Berry MA, Hargadon B, Shelley M, et al. Evidence of a role of tumor necrosis factor alpha in refractory asthma. N Engl J Med 2006;354:697-708.

13. Thomas PS, Heywood G. Effects of inhaled tumour necrosis factor alpha in subjects with mild asthma. Thorax 2002;57:774-8

14. Thomas PS, Yates DH, Barnes PJ. Tumor necrosis factor-alpha increases airway responsiveness and sputum neutrophilia in normal human subjects. Am J Respir Crit Care Med 1995;152:76-80.

15. Amrani Y, Martinet N, Bronner C. Potentiation by tumour necrosis factor-alpha of calcium signals induced by bradykinin and carbachol in human tracheal smooth muscle cells. Br J Pharmacol 1995;114:4-5.

16. Berry MA, Hargadon B, Shelley M, et al. Evidence of a role of tumor necrosis factor alpha in refractory asthma. N Engl J Med 2006;354:697-708.

17. Howarth PH, Babu KS, Arshad HS, et al. Tumour necrosis factor (TNFalpha) as a novel therapeutic target in symptomatic corticosteroid dependent asthma. Thorax 2005;60:1012-18.

18. Berry MA, Hargadon B, Shelley M, et al. Evidence of a role of tumor necrosis factor alpha in refractory asthma. N Engl J Med 2006;354:697-708.

19. Erin EM, Leaker BR, Nicholson GC, et al. The effects of a monoclonal antibody directed against tumor necrosis factor-alpha in asthma. Am J Respir Crit Care Med 2006;174:753-62.

20. Morjaria JB, Chauhan AJ, Babu KS, et al. The role of a soluble TNF $\alpha$ receptor fusion protein (etanercept) in corticosteroid refractory asthma: a double blind randomised, placebo controlled trial. Thorax 2008:63:584-91.

21. Berry MA, Hargadon B, Shelley M, et al. Evidence of a role of tumor necrosis factor alpha in refractory asthma. N Engl J Med 2006;354:697-708.

\section{Pleurodesis for malignant pleural effusion: talc, toxicity and where next?}

\section{Helen E Davies, ${ }^{1}$ Y C Gary Lee, ${ }^{1,2}$ Robert J 0 Davies $^{1}$}

Malignant pleural effusion accounts for $22 \%$ of all pleural effusions, and affects about 300000 patients annually (UK and

\footnotetext{
${ }^{1}$ Oxford Pleural Disease Unit, Oxford Centre for Respiratory Medicine and University of Oxford, Oxford, UK; ${ }^{2}$ Centre for Respiratory Research, University College London, London, UK
}

Correspondence to: Dr Robert J 0 Davies, Oxford Centre for Respiratory Medicine, Churchill Hospital, Oxford 0X3 7LJ, UK; robert.davies@ndm.ox.ac.uk
USA). Approximately $50 \%$ of patients with breast cancer, $25 \%$ of those with lung cancer and $>90 \%$ with pleural mesothelioma develop a symptomatic malignant pleural effusion. Thoracentesis provides effective short term symptomatic relief but most large malignant pleural effusions recur, ${ }^{1}$ and pleurodesis is then the standard treatment. A wide range of compounds have been used as pleurodesing agents, but talc is preferred by the majority of respiratory physicians worldwide. $^{2}$

The Medicines and Healthcare Products Regulatory Authority (MHRA) has recently completed an urgent review of the safety of talc as a pleurodesis agent for malignant effusion, reclassifying it as a medicinal product rather than medical device. ${ }^{3}$ This review requires that from January 2008, manufacturers must submit regulatory data if they wish their talc preparation to be used for pleurodesis. This is a milestone on the path towards improved care for malignant effusion as it heralds the first time an agent for intrapleural administration will be regulated under the systems used for biologically active drugs.

Talc is a magnesium silicate hydroxide $\left(\mathrm{Mg}_{3}\left(\mathrm{Si}_{2} \mathrm{O}_{5}\right)_{2}(\mathrm{OH})_{2}\right)$ and is mined, milled and sterilised prior to clinical use, although 